\title{
Resource Allocation in Hybrid Macro/Femto Networks ${ }^{1}$
}

\author{
Xiaoli $\mathrm{Chu}^{2}$, Yuhua Wu \\ Department of Electronic Engineering \\ King's College London \\ London WC2R 2LS, UK \\ E-mail: \{xiaoli.chu, yuhua.wu\}@kcl.ac.uk
}

\author{
Lamia Benmesbah, Wing-Kuen Ling \\ Centre for Digital Signal Processing Research \\ King's College London \\ London WC2R 2LS, UK \\ E-mail: \{lamia.benmesbah,wing-kuen.ling\}@kcl.ac.uk
}

\begin{abstract}
Femtocell has been considered by the wireless industry as a promising solution not only to improve indoor coverage, but also to unload traffic from overburdened macrocell networks. In hybrid macro/femto networks, macrocells may have to share the same spectrum with femtocells, due to spectrum availability and network infrastructure considerations, in spite of potentially excessive interference caused by densely deployed femtocells. In this paper, we propose a decentralized resource allocation scheme for the orthogonal frequency division multiple access (OFDMA) downlink of a shared-spectrum macro/femto network, where each femtocell randomly selects a subset of available OFDMA resources for transmission, in order to achieve decentralized inter-cell interference avoidance. The performance of the decentralized resource allocation scheme is evaluated by simulations based on per-cell or network-wide performance metrics. Simulation results provide insights on how the resource allocation in a shared-spectrum hybrid macro/femto network should take into account the spatial density of femtocells and indoor/outdoor radio propagation channel conditions.
\end{abstract}

Keywords-Femtocell, macrocell, OFDMA, resource block (RB), outage probability, areal spectral efficiency (ASE).

\section{INTRODUCTION}

Mobile user equipments (UEs) are becoming increasingly indispensable in our daily lives, but almost all current cellular networks are facing problems of imperfect coverage, especially indoors. One cost-effective solution for mobile operators to improve coverage is the emerging femtocell network, where femtocell access points (FAPs) [1] are overlaid on macrocells. Each FAP provides high-data-rate connections to UEs within a short range using the same radio-access technology as the macrocell underlay. Indoor femtocells can solve the problem of weak macrocell signals inside buildings and offload a large amount of traffic from outdoor macrocells. On the other hand, orthogonal frequency division multiple access (OFDMA) has been considered in the downlink for next generation wireless networks [2-3]. OFDMA-based femtocell is widely expected to deliver massive improvements in coverage and capacity [1].

Inter-cell interference management is among the most urgent challenges that operators must face before femtocells can be commercially deployed, given that most femtocells will be deployed within coverage of macrocells. As plug-and-play devices, most FAPs will be deployed by end users. The number and locations of active FAPs are thus by no means known to operators. Therefore, interference caused by femtocells cannot be managed using conventional network planning methods. Interference avoidance strategies [4], wherein femtocells avoid rather than suppress inter-cell interference, would be more likely to work. Furthermore, inter-cell interference in a hybrid macro/femto network depends largely on the femtocell access policy used, which defines how a femtocell allows or restricts its usage to users or UEs [4]. In this paper, we will focus on the closed-access femtocell, which serves a group of authorized UEs only, because closed access would be preferred by home subscribers of the femtocell [5].

To mitigate inter-cell interference, frequency reuse [6-8] and inter-cell coordination [9-10] schemes have been studied in OFDMA macro networks. A centralized downlink frequency planning across femto and macro cells was proposed in [11], but the very large number of femtocells may significantly complicate the centralized optimization process. The spectrum allocation policy in [12] avoids cross-tier interference by assigning orthogonal spectrum resources to the macro tier and the femto tier, and mitigates femto-to-femto interference by allowing each femtocell to access only a random subset of the spectrum resources that are assigned to the femto tier. However, many operators do not have enough spectra to assign a dedicated spectrum for femtocell deployments. Femtocells may have to operate in the same spectrum as macrocells due to spectrum availability and infrastructure considerations [11].

In this paper, we propose a decentralized resource allocation scheme for the OFDMA downlink of a shared-spectrum hybrid macro/femto network, in the effort to achieve decentralized inter-cell interference avoidance. OFDMA radio resources are partitioned in the time and frequency domains into resource blocks (RBs) [13]. In this resource allocation scheme, for each transmission time interval, a macrocell can use all the available RBs, while each femtocell can only randomly select a subset of the available RBs for transmission. As a result, the average number of interfering femtocells in each RB is reduced. How the size of the RB-subset should be decided with respect to the total number of available RBs per transmission time interval is studied based on per-cell or network-wide performance metrics, which are outage probability, spatial throughput [14], throughput per cell, and areal spectral efficiency [12]. The performance of the decentralized resource allocation scheme is evaluated through simulations.

The rest of the paper is organized as follows. The system model is described in Section II. The decentralized resource allocation scheme and performance metrics are presented in Section III. Simulation results and their interpretation are provided in Section IV. Conclusions are drawn in Section V.

\footnotetext{
${ }^{1}$ This work was supported in part by EPSRC CASE/CNA/07/106

${ }^{2}$ Corresponding author: Xiaoli Chu (xiaoli.chu@kcl.ac.uk)
} 


\section{SYSTEM MODEL}

We consider the OFDMA downlink of a shared-spectrum hybrid macro/femto network. The central hexagonal macrocell has a radius of $r_{\mathrm{M}}$. The macro base station (BS) $B_{0}$ is located in the middle of the macrocell. There are $U_{\mathrm{M}}$ outdoor macro UEs randomly distributed over the macrocell coverage area. For simplicity, co-channel interference from neighboring macrocell transmissions is ignored [15]. Closed-access femtocells are randomly overlaid on the macrocell. The locations of FAPs at a point in time form a stationary Poisson point process (SPPP) on $\mathrm{R}^{2}$ [14], with a spatial intensity of $\lambda_{\mathrm{F}}$, which gives the average number of femtocells per unit area. Each femtocell has a radius of $r_{\mathrm{F}}$ and serves $U_{\mathrm{F}}$ indoor UEs. If the mean number of femtocells per macrocell is given by $N_{\mathrm{F}}$, then the total number of UEs served by the hybrid macro/femto network is given by $U=U_{\mathrm{M}}+N_{\mathrm{F}} U_{\mathrm{F}}$

The basic resource unit that is addressable for OFDMA transmissions is an RB, which is a two-dimensional concept defined in the time-frequency space. In the 3GPP Release 8 [13], an RB has the time-duration of $1 \mathrm{~ms}$ and spans either 12 subcarriers with a subcarrier bandwidth of $15 \mathrm{kHz}$ or 24 subcarriers with a subcarrier bandwidth of $7.5 \mathrm{kHz}$. Intra-cell interference is avoided by maintaining orthogonality between co-cell UEs in OFDMA networks [12], [16], i.e., one scheduled UE per RB in each cell. Without loss of generality, we assume that a macro BS or an FAP assigns equal transmission power to all RBs for a given transmission time interval [12]. Since cellular networks are mostly interference limited, thermal noise at the receiver is neglected in this paper.

The downlink channel from a BS/FAP to a UE is composed of a possible deterministic wall-penetration loss component, a deterministic distance-dependent path-loss component (with a fixed path-loss exponent), and a random distance-independent component, which includes both slowly-varying lognormal shadowing and Rayleigh fast fading [14]. Independent and identically-distributed shadowing and fading statistics are assumed across all RBs for a given transmission time interval. The shadowing and fading coefficients are assumed to remain constant within each RB but may vary from one RB to another.

Accordingly, for a given $\mathrm{RB}$, the received signal-tointerference ratio (SIR) at a macro UE is given by

$$
\operatorname{SIR}_{\mathrm{M}}=\frac{P_{\mathrm{M}} A D_{\mathrm{M}}^{-\alpha_{\mathrm{M}}}}{\sum_{i \in \Phi} P_{i} \phi B_{i}\left|X_{\mathrm{Mi}}\right|^{-\alpha_{\mathrm{MF}}}}
$$

where $D_{\mathrm{M}}$ is the distance from the macro BS $B_{0}$ to the macro $\mathrm{UE},\left|X_{\mathrm{M} i}\right|$ is the distance from FAP $i$ to the macro UE, $\alpha_{\mathrm{M}}$ is the path loss exponent on the link from the macro BS to the macro $\mathrm{UE}, \alpha_{\mathrm{MF}}$ is the path loss exponent on the link from an FAP to the macro UE, $P_{\mathrm{M}}$ is the transmission power of the macro BS, $P_{i}$ is the transmission power of FAP $i, \phi$ represents wallpenetration loss, $A$ is the random channel gain from the macro BS to the macro UE, $B_{i}$ is the random channel gain from FAP $i$ to the macro UE, and $\Phi$ denotes the set of interfering FAPs.

For a given $\mathrm{RB}$, the received SIR of a femto UE is given by

$$
\operatorname{SIR}_{\mathrm{F}}=\frac{P_{\mathrm{F}} G r_{\mathrm{F}}^{-\alpha_{\mathrm{F}}}}{P_{\mathrm{M}} \phi Q D_{\mathrm{F}}^{-\alpha_{\mathrm{FM}}}+\sum_{i \in \Phi} P_{i} \phi^{2} H_{i}\left|X_{\mathrm{Fi}}\right|^{-\alpha_{\mathrm{FF}}}}
$$

where $D_{\mathrm{F}}$ is the distance from the macro BS to the femto UE, $\left|X_{\mathrm{F} i}\right|$ is the distance from an interfering FAP $i$ to the femto UE, $\alpha_{\mathrm{F}}$ is the path loss exponent on the link from the home FAP to the femto UE, $\alpha_{\mathrm{FM}}$ is the path loss exponent on the link from the macro BS to the femto UE, $\alpha_{\mathrm{FF}}$ is the path loss exponent on the link from an interfering FAP to the femto UE, $P_{\mathrm{F}}$ is the transmission power of the home FAP, $G$ is the channel gain from the home FAP to the femto $\mathrm{UE}, Q$ is the channel gain from the macro BS to the femto $\mathrm{UE}$, and $H_{i}$ is the channel gain from an interfering FAP $i$ to the femto UE. We consider the worst case that all femto UEs are located on the edge of their home femtocell. Double wall-penetration losses are assumed for all links from interfering FAPs to the femto UE of interest.

In (1) and (2), by defining different path-loss exponents (i.e., $\alpha_{\mathrm{M}}, \alpha_{\mathrm{MF}}, \alpha_{\mathrm{F}}, \alpha_{\mathrm{FM}}$ and $\alpha_{\mathrm{FF}}$ ), different random channel gains (i.e., $A, B_{i}, G, Q$ and $H_{i}$ ) and the wall-penetration loss factor (i.e., $\phi$ ), different radio propagation characteristics of outdoor and indoor channels are taken into account.

\section{Resource Allocation In MACro/FEMto Networks}

\section{A. Decentralized Resource Allocation}

In OFDMA networks, inter-cell interference can be avoided from a perspective of temporal and spectral reuse of radio resources in different cells. For a shared-spectrum hybrid macro/femto network that employs OFDMA in the downlink, we propose a decentralized resource allocation scheme that can provide randomized interference avoidance. Given that there are $F$ distinct RBs in total for each transmission time interval, we allow a macro BS to use all the $F$ RBs for its downlink transmissions, but an FAP to use only $K(\leq F)$ RBs, where both $F$ and $K$ are positive integers. For ease of implementation, the value of $K$ is kept the same for all femtocells. Accordingly, the fraction of radio resources per transmission interval accessible by each femtocell is given by

$$
\rho_{\mathrm{F}}=\frac{K}{F}
$$

where $0<\rho_{\mathrm{F}} \leq 1$ by definition. In the special case of $\rho_{\mathrm{F}}=1$, each femtocell has access to all the available RBs.

If each femtocell chooses the $K$ distinct RBs independently and with equal probability, the probability of a femtocell selecting a given $\mathrm{RB}$ for transmission is given by

$$
\operatorname{Pr}=\rho_{\mathrm{F}}
$$

which shows that the average number of interfering femtocells in each RB is effectively reduced, mitigating both femto-tomacro and femto-to-femto interference.

Therefore, the set $\Phi$ in (1) and (2) is actually the set of FAPs that have access to the given RB. At a point in time, the set $\Phi$ forms a marked SPPP and has a spatial intensity of $\lambda_{\mathrm{F}} \rho_{\mathrm{F}}\left(\leq \lambda_{\mathrm{F}}\right)$.

\section{B. Performance Metrics}

Performance of the decentralized resource allocation scheme is evaluated based on four different per-cell or network-wide performance metrics, which are able to effectively show how the probability of each femtocell transmitting in a given RB, 
i.e., $\rho_{\mathrm{F}}$, affects the performance per cell or the performance of the whole hybrid macro/femto network.

Under the assumptions made in Section II and Section III-A, the outage probability of a macro UE and of a femto UE is given respectively by

$$
\begin{gathered}
q_{\mathrm{M}}\left(\rho_{\mathrm{F}}\right)=\operatorname{Pr}\left(\operatorname{SIR}_{\mathrm{M}}<\gamma_{\mathrm{M}}\right) \\
q_{\mathrm{F}}\left(\rho_{\mathrm{F}}\right)=\operatorname{Pr}\left(\operatorname{SIR}_{\mathrm{F}}<\gamma_{\mathrm{F}}\right)
\end{gathered}
$$

where $\gamma_{\mathrm{M}}$ and $\gamma_{\mathrm{F}}$ are the SIR requirements of a macro UE and a femto UE, respectively.

Based on the definition of outage probability, spatial throughput is defined as the expected spatial density of successful transmissions [14]. The spatial throughput of the hybrid macro/femto network, subject to SIR requirements of $\gamma_{M}$ and $\gamma_{\mathrm{F}}$, is given by

$$
\tau\left(\rho_{\mathrm{F}}\right)=\mu_{\mathrm{M}}\left[1-q_{\mathrm{M}}\left(\rho_{\mathrm{F}}\right)\right]+\lambda_{\mathrm{F}} \rho_{\mathrm{F}}\left[1-q_{\mathrm{F}}\left(\rho_{\mathrm{F}}\right)\right]
$$

where $\mu_{\mathrm{M}}$ is the spatial density of macro UEs that have access to a given RB.

If adaptive modulation with $L(>0)$ discrete rates is adopted, then when the received SIR lies in $\left[\Gamma_{l}, \Gamma_{l+1}\right)$, for $l \in\{1, \ldots, L\}$, the $\mathrm{BS}$ or FAP decides the transmission rate according to

$$
b_{l}=\log _{2}\left(1+\frac{\Gamma_{l}}{\Psi}\right), \quad(\mathrm{b} / \mathrm{s} / \mathrm{Hz})
$$

where $\Psi$ is the Shannon gap for QAM modulations [12].

If each UE feedbacks the instantaneous SIR in a given RB to its home BS or FAP, i.e., impact of imperfect feedback and/or channel estimation is not considered, then the expected throughput per cell (in b/s/Hz) [12] is given by

$$
\begin{gathered}
T_{\mathrm{M}}=\sum_{l=1}^{L-1} b_{l} \operatorname{Pr}\left(\Gamma_{l} \leq \operatorname{SIR}_{\mathrm{M}}<\Gamma_{l+1}\right)+b_{L} \operatorname{Pr}\left(\operatorname{SIR}_{\mathrm{M}} \geq \Gamma_{L}\right) \\
T_{\mathrm{F}}=\rho_{\mathrm{F}}\left[\sum_{l=1}^{L-1} b_{l} \operatorname{Pr}\left(\Gamma_{l} \leq \operatorname{SIR}_{\mathrm{F}}<\Gamma_{l+1}\right)+b_{L} \operatorname{Pr}\left(\operatorname{SIR}_{\mathrm{F}} \geq \Gamma_{L}\right)\right]
\end{gathered}
$$

The areal spectral efficiency (ASE) (in $\mathrm{b} / \mathrm{s} / \mathrm{Hz} / \mathrm{m}^{2}$ ) of the hybrid macro/femto network is then given by

$$
\mathrm{ASE}=\mu_{\mathrm{M}} T_{\mathrm{M}}+\lambda_{\mathrm{F}} T_{\mathrm{F}}
$$

\section{Simulation Results}

We perform simulations based on the performance metrics defined in Section III-B, in order to study how the fraction of radio resources accessible by each femtocell per transmission time interval should be determined in the decentralized resource allocation scheme.

In the simulations, the spatial distributions of outdoor macro UEs and indoor femto UEs on the $\mathrm{R}^{2}$ plane are obtained by independently generating each $D_{\mathrm{M}}$ or $D_{\mathrm{F}}$ following a uniform distribution in the range of $\left[1, r_{\mathrm{M}}\right]$, where we assume that all UEs and FAPs are at least 1 meter away from the central macro BS; and the angular direction of each UE or FAP with respect to the central macro $\mathrm{BS}$ is independently generated following a uniform distribution in $(0,2 \pi]$. Without loss of generality, we assume all FAPs have the same transmission power $P_{\mathrm{F}}$, and the Rayleigh fast fading has a unit average power. In the calculations for (9) to (11), we set $b_{l}=l$ and $\Gamma_{l}=$ $\Psi\left(2^{l}-1\right)$, for $l=1, \ldots, L$. The values of major system parameters used in the simulations are given in Table I.

Fig. 1 and Fig. 2 show the outage probability versus $\rho_{\mathrm{F}}$, for $N_{\mathrm{F}}=50,100$, and 200. In Fig. $1, \phi=5 \mathrm{~dB}, \alpha_{\mathrm{MF}}=\alpha_{\mathrm{FF}}=4$, representing a relatively high-attenuation channel, and $r_{\mathrm{M}}=$ 1000 m. In Fig. $2, \phi=2 \mathrm{~dB}, \alpha_{\mathrm{MF}}=\alpha_{\mathrm{FF}}=3.5$, representing a low-attenuation channel, and $r_{\mathrm{M}}=300 \mathrm{~m}$. In both figures, we can observe that the outage probabilities of macro UEs and femto UEs increase with the increase of $\rho_{\mathrm{F}}$ and $N_{\mathrm{F}}$. This is because the interference caused by femtocells becomes severer when each femtocell accesses a larger fraction of the available RBs and with a higher spatial intensity of femtocells. In order to maintain a specific outage probability for a macro UE or a femto UE, a reduced $\rho_{\mathrm{F}}$ has to be used for a larger $N_{\mathrm{F}}$.

TAble I. System Parameters of the Simulations

\begin{tabular}{|l|l|}
\hline \multicolumn{1}{|c|}{ System Parameters } & Values \\
\hline Macrocell outdoor path-loss exponent $\left(\alpha_{\mathrm{M}}\right)$ & 4 \\
Femtocell indoor path-loss exponent $\left(\alpha_{\mathrm{F}}\right)$ & 3 \\
Macro BS to femto UE path-loss exponent $\left(\alpha_{\mathrm{FM}}\right)$ & $=\alpha_{\mathrm{M}}$ \\
Femto-to-femto path-loss exponent $\left(\alpha_{\mathrm{FF}}\right)$ & 3.5 or 4 \\
FAP to macro UE path-loss exponent $\left(\alpha_{\mathrm{MF}}\right)$ & $=\alpha_{\mathrm{FF}}$ \\
Wall-penetration loss $(\phi)$ & 2 or $5 \mathrm{~dB}$ \\
Macrocell outdoor lognormal shadowing sd $\left(\sigma_{\mathrm{M}}\right)$ & $8 \mathrm{~dB}$ \\
Femtocell indoor lognormal shadowing sd $\left(\sigma_{\mathrm{F}}\right)$ & $4 \mathrm{~dB}$ \\
Femto-to-femto lognormal shadowing sd $\left(\sigma_{\mathrm{FF}}\right)$ & $12 \mathrm{~dB}$ \\
Femto-to-macro lognormal shadowing sd $\left(\sigma_{\mathrm{MF}}\right)$ & $10 \mathrm{~dB}$ \\
Macro-to-femto lognormal shadowing sd $\left(\sigma_{\mathrm{FM}}\right)$ & $10 \mathrm{~dB}$ \\
Macro BS transmission power $\left(P_{\mathrm{M}}\right)$ & $43 \mathrm{dBm}$ \\
FAP transmission power $\left(P_{\mathrm{F}}\right)$ & $20 \mathrm{dBm}$ \\
Number of outdoor macro UEs per macrocell $\left(U_{\mathrm{M}}\right)$ & 200 \\
Number of indoor UEs per femtocell $\left(U_{\mathrm{F}}\right)$ & 2 \\
Femtocell coverage radius $\left(r_{\mathrm{F}}\right)$ & $30 \mathrm{~m}$ \\
SIR requirement of a macro UE $\left(\gamma_{\mathrm{M}}\right)$ & $7 \mathrm{~dB}$ \\
SIR requirement of a femto UE $\left(\gamma_{\mathrm{F}}\right)$ & $15 \mathrm{~dB}$ \\
Shannon gap $(\Psi)$ & $3 \mathrm{~dB}$ \\
Number of adaptive modulation levels $(L)$ & 8 \\
\hline
\end{tabular}

In Fig. 1, for each given value of $N_{\mathrm{F}}$, a macro UE experiences a much higher outage probability than a femto $\mathrm{UE}$, indicating that the femto-to-femto interference is not as significant as the femto-to-macro interference. This is due to the double wall-penetration losses with $\phi=5 \mathrm{~dB}$ between neighboring femtocells. In Fig. 2, with a much shorter radius of the macrocell, for the same values of $N_{\mathrm{F}}$ as in Fig. 1, the spatial intensities of femtocells become much higher. The high femtocell density combined with the low-attenuation channel contributes to a significant increase of interference from femtocells, leading to unacceptably high outage probabilities of both macro and femto UEs.

Fig. 3 and Fig. 4 plot the spatial throughput versus $\rho_{\text {F }}$, under the same conditions as in Fig. 1 and Fig. 2, respectively. Fig. 3 shows that the spatial density of successful transmissions in the 
hybrid macro/femto network increases with the increase of $\rho_{\mathrm{F}}$ and $N_{\mathrm{F}}$, under a high-attenuation channel and with relatively low spatial densities of femtocells. In Fig. 4, when the channel is low-attenuation and the spatial densities of femtocells are high, we observe that for any given value of $\rho_{\mathrm{F}}$, the spatial throughput decreases with the increase of $N_{\mathrm{F}}$. For larger values of $N_{\mathrm{F}}$, the spatial throughput decreases with $\rho_{\mathrm{F}}$ when $\rho_{\mathrm{F}}$ goes beyond a certain value.

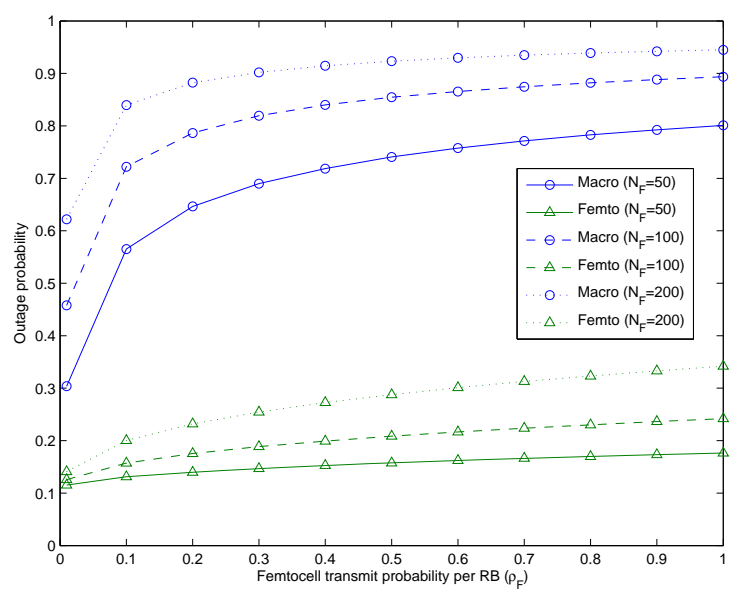

Fig. 1 Outage probability vs. $\rho_{\mathrm{F}}$, for $\phi=5 \mathrm{~dB}, \alpha_{\mathrm{FF}}=4$, and $r_{\mathrm{M}}=1000 \mathrm{~m}$.

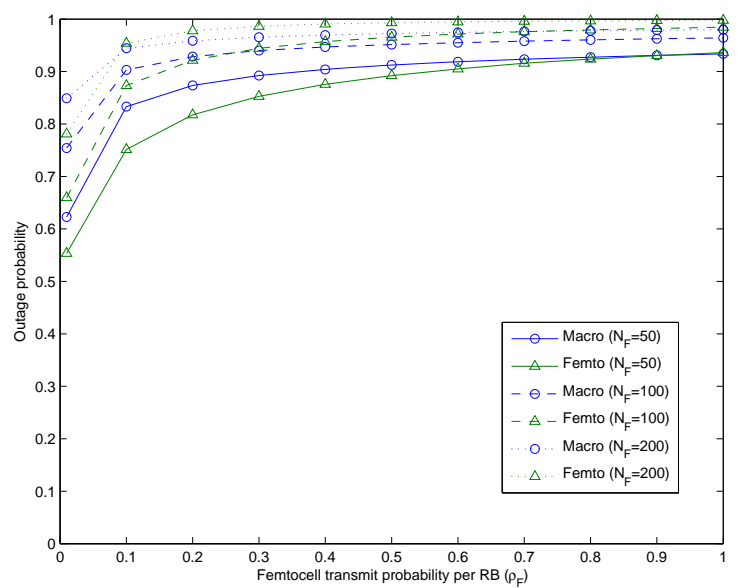

Fig. 2 Outage probability vs. $\rho_{\mathrm{F}}$, for $\phi=2 \mathrm{~dB}, \alpha_{\mathrm{FF}}=3.5$, and $r_{\mathrm{M}}=300 \mathrm{~m}$.

Fig. 5 and Fig. 6 show the throughput per cell versus $\rho_{\mathrm{F}}$, under the same conditions of Fig. 1 and Fig. 2, respectively. Both figures show that the throughput per macrocell decreases as $\rho_{\mathrm{F}}$ increases, while the throughput per femtocell increases with the increase of $\rho_{\mathrm{F}}$. This confirms that as each femtocell is allowed to access only a reduced fraction of available RBs, the femto-to-macro interference is reduced and the throughput per macrocell increases. However, if the number of accessible RBs per transmission interval is made too small for each femtocell, the throughput per femtocell becomes unsatisfactory. For both macrocell and femtocells, the throughput per cell decreases with the increase of $N_{\mathrm{F}}$. In order to maintain a specific throughput per macrocell, a reduced $\rho_{\mathrm{F}}$ has to be used for a larger $N_{\mathrm{F}}$. By comparing Fig. 6 with Fig. 5 , we can see that the throughput per cell is significantly reduced under a lowerattenuation channel and higher spatial densities of femtocells, indicating its sensitivity to the radio propagation channel and the density of femtocells.

Fig. 7 and Fig. 8 plot the ASE versus $\rho_{\text {F, }}$ under the same conditions as in Fig. 1 and Fig. 2, respectively. Fig. 7 shows that the ASE of the hybrid macro/femto network increases with the increase of $\rho_{\mathrm{F}}$ and $N_{\mathrm{F}}$, under a high-attenuation channel and with low spatial densities of femtocells. In Fig. 8, when the channel is low-attenuation and the femtocell densities are high, we can see that for large values of $\rho_{\mathrm{F}}$, the ASE decreases with the increase of $N_{\mathrm{F}}$, indicating that employing more femtocells does not improve spatial reuse. For $N_{\mathrm{F}} \geq 200$, the ASE stops increasing with $\rho_{\mathrm{F}}$ when $\rho_{\mathrm{F}}$ goes beyond a certain value.

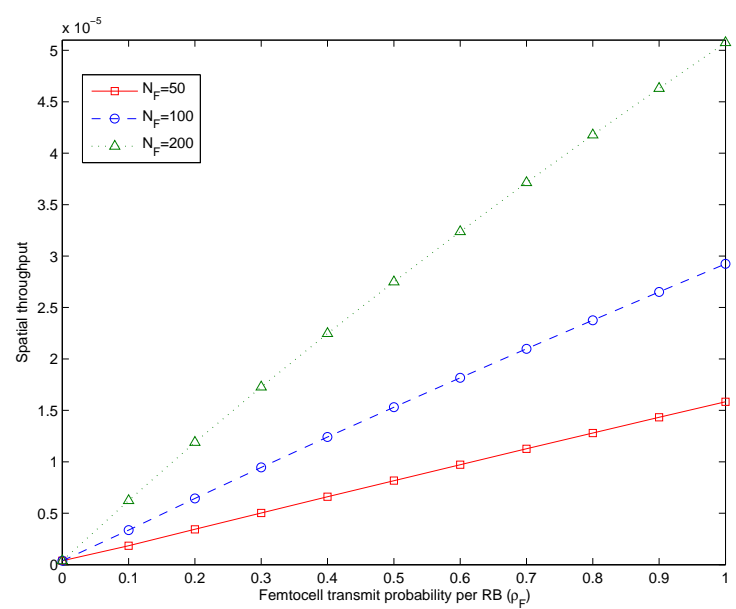

Fig. 3 Spatial throughput vs. $\rho_{\mathrm{F}}$, for $\phi=5 \mathrm{~dB}, \alpha_{\mathrm{FF}}=4$, and $r_{\mathrm{M}}=1000 \mathrm{~m}$.

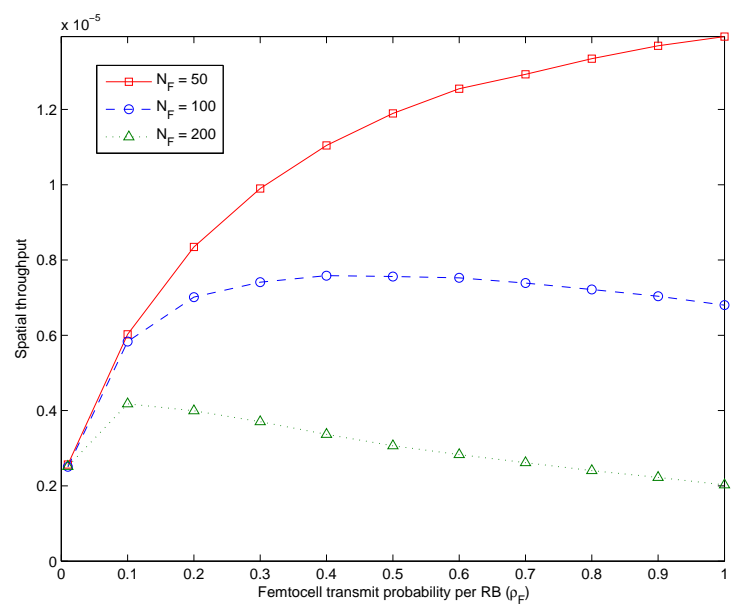

Fig. 4 Spatial Throughput vs. $\rho_{\mathrm{F}}$, for $\phi=2 \mathrm{~dB}, \alpha_{\mathrm{FF}}=3.5$, and $r_{\mathrm{M}}=300 \mathrm{~m}$.

\section{CONCLUSIONS}

In this paper, we have proposed a decentralized resource allocation scheme for the OFDMA downlink of a sharedspectrum hybrid macro/femto network. Simulation results have shown that by restricting each femtocell to access only a randomly selected subset of the available RBs per transmission time interval, decentralized inter-cell interference avoidance can be achieved. In order to maintain a spcific performance per cell or network-wide, each femtocell may have to access a decreasing fraction of the available RBs with an increasing 
femtocell density. Furthermore, the appropriate design of the decentralized resource allocation scheme for a macro/femto network needs to account the spatial intensity of femtocells and indoor/outdoor radio propagation characteristics.

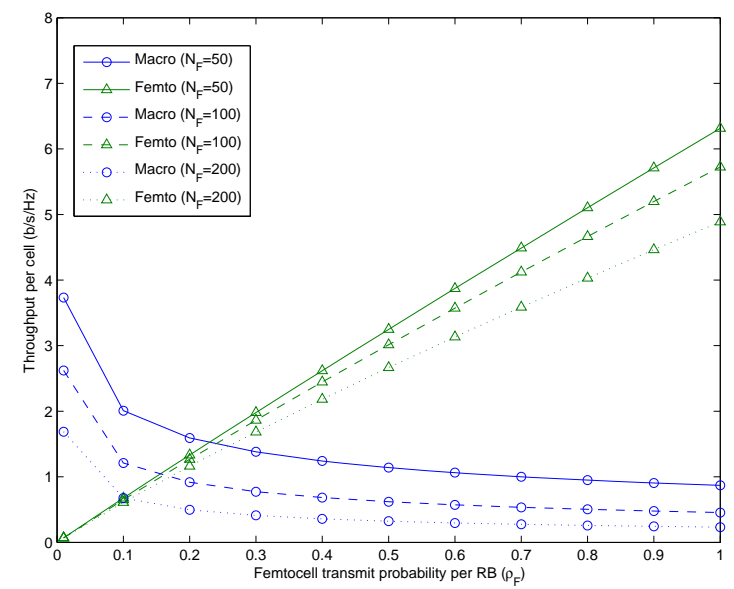

Fig. 5 Throughput per cell vs. $\rho_{\mathrm{F}}$, for $\phi=5 \mathrm{~dB}, \alpha_{\mathrm{FF}}=4$, and $r_{\mathrm{M}}=1000 \mathrm{~m}$.

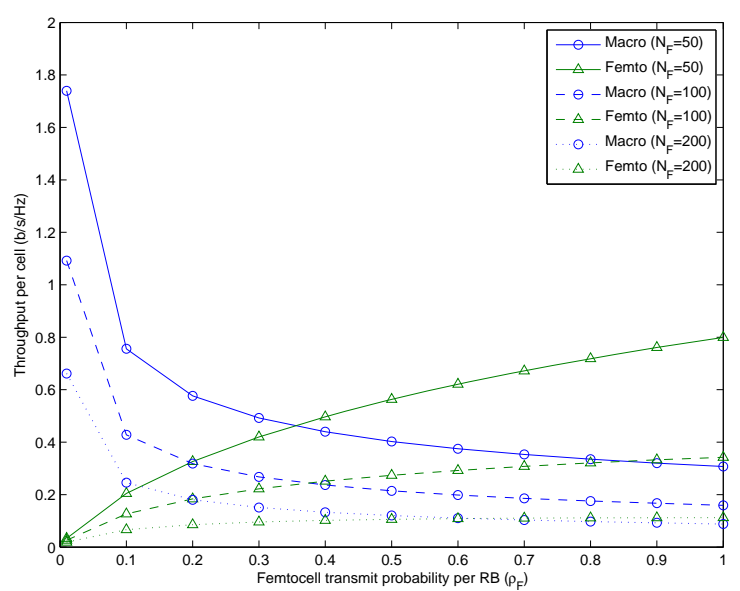

Fig. 6 Throughput per cell vs. $\rho_{\mathrm{F}}$, for $\phi=2 \mathrm{~dB}, \alpha_{\mathrm{FF}}=3.5$, and $r_{\mathrm{M}}=300 \mathrm{~m}$.

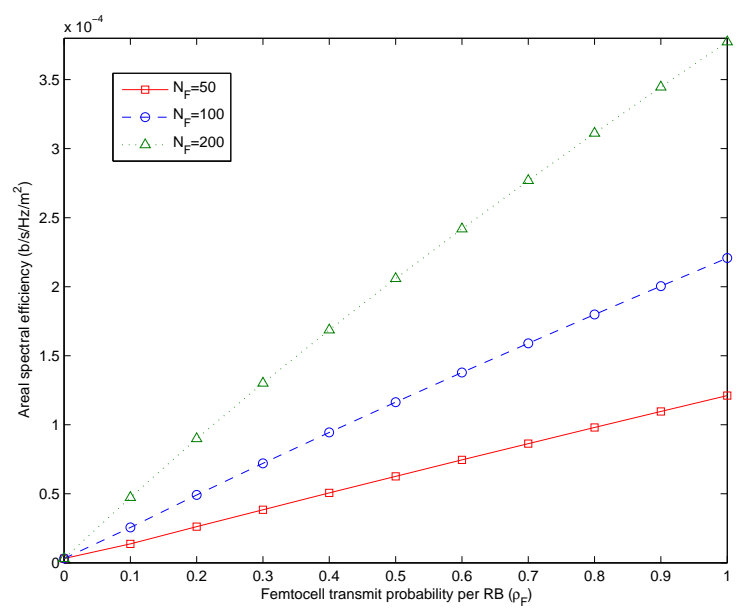

Fig. 7 ASE vs. $\rho_{\mathrm{F}}$, for $\phi=5 \mathrm{~dB}, \alpha_{\mathrm{FF}}=4$, and $r_{\mathrm{M}}=1000 \mathrm{~m}$.

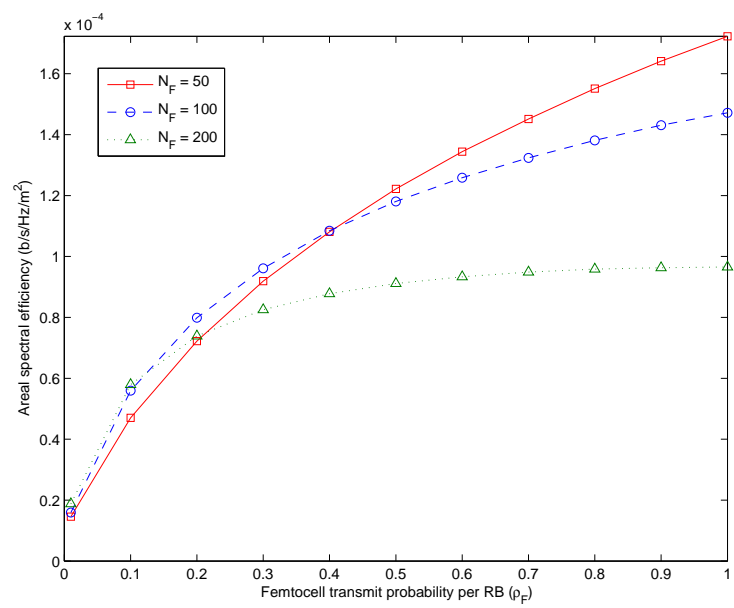

Fig. 8 ASE vs. $\rho_{\mathrm{F}}$, for $\phi=2 \mathrm{~dB}, \alpha_{\mathrm{FF}}=3.5$, and $r_{\mathrm{M}}=300 \mathrm{~m}$.

\section{REFERENCES}

[1] S. Yeh, S. Talwar, S. Lee, and H. Kim, "WiMAX femtocells: a perspective on network architecture, capacity, and coverage," IEEE Communications Magazine, vol. 46, iss. 10, pp. 58 - 65, Oct 2008.

[2] 3GPP LTE, http://www.3gpp.org/Highlights/LTE/LTE.htm.

[3] IEEE 802.16m-07/002r4, "TGm System Requirements Document (SRD)," approved 19 Oct 2007.

[4] V. Chandrasekhar, J. Andrews, and A. Gatherer, "Femtocell networks: a survey," IEEE Communications Magazine, vol. 46, iss. 9, pp. 59 - 67, Sep 2008.

[5] M. Latham, "Consumer attitudes to femtocell enabled in-home services - insights from a European survey," Femtocells Europe 2008, London June 2008.

[6] A. Stolyar and H. Viswanathan, "Self-organizing dynamic fractional frequency reuse in OFDMA systems," IEEE Conference on Computer Communications, pp. 691-699, 13-18 April 2008.

[7] S. E. Elayoubi, O. Ben Haddada, and B. Fourestie, "Performance evaluation of frequency planning schemes in OFDMA-based networks," IEEE Transactions on Wireless Communications, vol. 7, iss. 5, pp.16231633, May 2008.

[8] M. Assaad and A. Mourad, "New frequency-time scheduling algorithms for 3GPP/LTE-like OFDMA air interface in the downlink," IEEE VTC Spring 2008, pp. 1964 - 1969, 11-14 May 2008.

[9] M. C. Necker, "Local interference coordination in cellular OFDMA networks," IEEE VTC(F)’07, pp. 1741 - 1746, Sep 2007.

[10] A.Simonsson, "Frequency reuse and intercell interference co-ordination in E-UTRA," IEEE VTC(S)’07, pp.3091-3095, Apr 2007.

[11] D. Lopez-Perez, G. de la Roche, A. Valcarce, A. Juttner, and J. Zhang, "Interference avoidance and dynamic frequency planning for WiMAX femtocells networks," IEEE International Conf. on Commun. Systems, pp. 1579-1584, Nov 2008.

[12] V. Chandrasekhar and J. Andrews, "Spectrum allocation in two-tier networks," available at http://arxiv.org/abs/0805.1226.

[13] 3GPP Release 8, http://www.3gpp.org/Release-8.

[14] S. Weber, J. Andrews, and N. Jindal, "The effect of fading, channel inversion, and threshold scheduling on ad hoc networks," IEEE Trans. on Info. Theory, vol. 53, no. 11, pp. 4127 - 4149, Nov 2007.

[15] V. Chandrasekhar, J. Andrews, T. Muharemovic, Z. Shen, and A. Gatherer, "Power control in two-tier femtocell networks," IEEE Trans. on Wireless Commun., vol. 8, no. 8, pp. 4316 - 4328, Aug 2009.

[16] J. Andrews, A. Ghosh, and R. Muhamed, Fundamentals of WiMAX: Understanding Broadband Wireless Networking, Prentice Hall, 2007. 